IJP 00684

\title{
In situ jejunal uptake from liposomal systems
}

\author{
D.L. Schwinke, M.G. Ganesan and N.D. Weiner \\ College of Pharmacy. The University of Michigan, Ann Arbor, MI 48109 (U.S.A)
}

(Received November 10th, 1983)

(Modified version received January 17th. 1984)

(Accepted February 6th, 1984)

\section{Summary}

The in situ uptake from the rat jejunum of liposomes prepared fr om various saturated phosphatidylcholines in the presence or absence of choleste ol was determined. Liposomes prepared from distearoylphosphatidylcholine, with or without cholesterol, were taken up at the fastest rate (apparent first-order uptahe of intact liposomes with a half-life of about $15 \mathrm{~min}$ ). The lipid components of liposomes could not be found in compartments where liposomes would be expected to accumulate, i.e. liver, spleen. lymph nodes, or thoracic lymphatic duct. Furthermore. inulin, when encapsulated in the liposomes, could not be found in the urine. When either PEG-4000 (a non-absorbable marker) or glucose (an absorbable ntarker) was entrapped within the liposome, all of the liposomes and markers that we taken up still remained associated with the jejunum and the liposomes remained intact.

\section{Introduction}

The oral route of administration has the advantage of convenience. versatility, and safety. Many drugs cannot be administered orally due to chemical ir stability or poor absorption from the gastrointestinal tract. Recent studies have suggested that liposomes may be able to facilitate the gastrointestinal absorption o: drugs by protecting labile compounds from degradation, or by enhancing the uptake of poorly absorbed compounds (Patel and Ryman, 1976; Dapergolas and (iregoriadis, 1977). The broad spectrum of liposomal properties, together with the abil ty of many

Correspondence: N.D. Weiner, College of Pharmacy, University of Michigan, Ann Arbır. MI 48109. U.S.A. 
compounds to affect these properties renders the investigation of liposomes as an oral dosage form a complex and difficult task.

We have therefore attempted to isolate and systematically study a number of important parameters which have been reported to affect the uptake and disposition of drugs entrapped in liposomes.

(a) Lipid composition of the liposome. Since liposomes containing unsaturated and/or negatively charged lipids have been shown to be unstable in the presence of physiological concentrations of bile salts (Rowland and Woodley, 1980) and positively charged lipids are cytotoxic (Layton et al., 1980), we have concentrated our efforts on liposomes containing various saturated phosphatidylcholines in the presence and ahsence of cholesterol.

(b) Entrupment of markers in the liposome. The mechanism of uptake of liposomally entrapped drugs has not been established (Pagano, et al., 1981). Furthermore, there is considerable controversy as to whether liposomes themselves are able to Iraverse the gastrointestinal tract (Deshmukh. et al., 1981; Rowland and Woodley. 19811 . Wo have therefore studied the uptake and fate of empty liposomes as well as liposomen containing non-absorbable. non-metabolizable markers (inulin and PEG$40001)$ and liposomes containing a readily absorbable and metabolizable marker (gluconce).

(c) Comernlled area and enironment of uptake. These studies were carried out by a modilied in situ Doluisio Method (Komiya, et al.. 1980) so that the following parameters werc easily accessible to experimentation: (1) sampling from the intestinial lumen for hoth liposomes and markers; (2) dr ermination of uptake from a defincel section of the small intestine: and (3) determination of intrinsic uptake by eliminating environmental factors that can destabilize the liposomes, e.g. low $\mathrm{pH}$ valuen, bile valts. and phospholipases.

\section{Experiment:il}

\section{Materiesls}

The foll wing lipids were purchased from Sigma Chemicals (St. Louis, MO): dimyristoy/phosphatidylcholine (DMPC). dipalmitoylphosphatidylcholine (DPPC), distearov/phosphatidylcholine (DSPC). and cholesterol (CHOL). Cholesterol was purilicd by recrystallizing 3 times from ethanol solution and all other lipids were used in supplied, and stored below $0^{\circ} \mathrm{C}$. Thin-layer chromatography of all lipids revialsol a single spot. All other chemicals were reagent grade.

The following radiolahelled lipid and aqueous markers were purchased from Amerhitm (Arlington Heights, IL): I- $\alpha$-phosphatidylcholine di[1-14 C]palmitoyl $\left(\left.\right|^{1+}\left(C_{1}\right) P P(), \quad 1-\alpha-p h o s p h a t i d y l c h o l i n e ~ d i\left[1-{ }^{14} \mathrm{C}\right]\right.$ stearoyl $\left(\left[{ }^{14} \mathrm{C}\right] \mathrm{DSPC}\right)$. ['H]chulinterol and ['H]inulin. Mass balance experiments also utilized the following radiolabulled compounds purchased from New England Nuclear (Boston. MA): $\left.\left[1,2-{ }^{14} \mathrm{C}\right] \mathrm{Pl}: \mathrm{G}-\left.4000 \mathrm{and}\right|^{14} \mathrm{C}\right] \mathrm{glucose}$.

\section{Preperations of lipexsomes}

Phospholipids ( $32 \mu \mathrm{mol})$, with or without cholesterol $(16 \mu \mathrm{mol})$, were dried from a 
chloroform solution at room temperature under nitrogen to form a thin tilm on the sides of a $20-\mathrm{ml}$ glass vial. Residual chloroform was removed by placing t/te vial in a vacuum for at least $12 \mathrm{~h}$. The isotonic phosphate-buffered saline (PBS) us ed in these studies consisted of $67 \mathrm{mM}$ sodium phosphate adjusted to $\mathrm{pH} 6.0$ with sodium hydroxide.

Liposomes were formed by adding $1.0 \mathrm{ml}$ of PBS to the dried lipid via s followed by incubation for $30 \mathrm{~min}$ in a water bath at a temperature $2-3^{\circ} \mathrm{C}$ above the phase transition temperature of the phospholipid component. Compounds whith were to be entrapped were dissolved in PBS before being added to the vials con aining the dried lipids. Dispersion of the lipids was aided by agitation in a vortex mi) er for 3-5 min during the incubation step. The vials were then sonicated for $30 \mathrm{~s}$ in a bath-type sonicator (Branson Cleaning Equipment, Shelton, $C N$ ) at the same temf erature as the incubation, and allowed to equilibrate at room temperature for at lea $14 \mathrm{~h}$.

Free and entrapped aqueous markers were separated by passing the liposome suspension through a $1 \times 45 \mathrm{~cm}$ column of Sephadex G-50 (coarse) (I'harmacia, Piscalaway, NJ). Liposomes eluted (with fresh buffer) immediately afte the void volume and the first $3 \mathrm{ml}$ of this fraction were collected and further ' $u$ ashed' by centrifugation 3 times for $8 \mathrm{~min}$ at $8000 \mathrm{rpm}$. Each time the supern stant was removed and the liposome pellet resuspended in 2-4 $\mathrm{ml}$ of buffer. Lipo iomes not containing entrapped markers, i.e. 'enty' liposomes, were also subje it to this procedure.

\section{Scanning transmission electron microscopy (STEM)}

Empty liposomes and liposomes containing PEG-4000 were examined under the STEM (Jeol, Tokyo, Japan) by a procedure similar to that described by niyamoto and Stoeckenius (1971) in order to characterize the preparations and to de termine if the entrapment of certain macromolecules produced any gross structura changes. All liposomal preparations tested appeared multilamellar and heterodisper ied in size (with a mean diameter of $(1.2 \mu \mathrm{m}$ and a particle size range of $0.08-0.3 \mu \mathrm{m}$ ). The liposomes appeared to be unaffected by entrapment of any of the marker used.

\section{In situ rat intestinal uptake}

A rnodified in situ Doluisio Method (Komiya, et al.. 1980) was employe $d$ in these studies. Male Sprague-Dawley rats, fasted overnight. weighing between $250-300 \mathrm{~g}$ were anesthetized with $20 \%$ ethylcarbamate (Sigma Chemicals, St. Lo:dis, MO) solution by two $1-\mathrm{ml}$ intraperitoneal injections $15 \mathrm{~min}$ apart. A midline abdominal incision was made from which the small intestine could be exposed. The proximal end of the jejunum was first cannulated with L-shaped tubing $(4 \mathrm{~mm} 0.1 . ; 2 \mathrm{~mm}$ i.d.). A second cannula was then placed $22 \mathrm{~cm}$ distal to the first and the seg ment was rinsed with $0.9 \%$ sodium chloride at $37^{\circ} \mathrm{C}$ until clear. The segment was ar anged in $\mathrm{S}$-shaped curves free of constrictions, covered with gauze, and kept moist "vith $0.9 \%$ sodium chloride solution at $37^{\circ} \mathrm{C}$. the cannulas were attached to the harrels of plastic, disposable 5-ml syringes (Becton Dickenson, Rutherford, NJ) whic/t were, in turn, connected to a 2-directional piston air pump (Fluid Meter, Oyster Fay, NY).

The jejunal segment was given a final rinse of PBS before the sta $t$ of the 
experiment. Two $\mathrm{ml}$ of liposome suspension or PBS solution were introduced into the lumen of the segment and oscillated back and forth twice before taking the 'zero-time' sample. The fluid was pumped at $0.075 \mathrm{ml} / \mathrm{s}$ and the direction was reversed approximately every $10 \mathrm{~s}$ so that not more than $0.75 \mathrm{ml}$ were displaced from the segment at any given time. Sample volumes of $25 \mu$ l were taken from the distal cannula every $5 \mathrm{~min}$ for $30 \mathrm{~min}$.

No significant water flux into or out of the jejunum was detected in experiments using trace amounts of $\left[{ }^{3} \mathrm{H}\right] \mathrm{PEG}$ in PBS. On occasion, some liposome experiments did exhibit a high degree of water loss from the jejunum. This was usually associated with visible signs of trauma, i.e. restricted blood flow or bleeding at the site of cannulation. The results of such experiments were not used.

A second difficulty which was encountered in the uptake studies was the visible aggregation or 'clumping' of the liposome suspension during the experiment. This phenomenon appeared to be due to excess mucous of the jejunum, or to secretions as a result of tissue damage at the site of cannulation. When visible clumping of the liposomes was observed, the experiment was discarded.

\section{Tissue sampling}

\section{Tissue solubilization studies}

In situ uptake studies using empty DSPC-CHOL $(2: 1)$ liposomes with $\left[{ }^{14} \mathrm{C}\right] \mathrm{DSPC}$ were performed as described earlier. At the end of each experiment the liposome suspension was removed and replaced with $2 \mathrm{ml}$ of PBS. After $1 \mathrm{~h}$ the rats were sacrificed and the liver, spleen, lymph nodes, and thoracic lymph duct were removed. The liver and spleen were lightly rinsed and excess water was removed with filter paper. Samples of between 100-200 $\mathrm{mg}$ were taken from the liver and spleen. whereas the entire lymph nodes or ducts $(17-275 \mathrm{mg})$ were used.

Tissue samples were placed in $20 \mathrm{ml}$ scintillation vials with plastic-lined screw caps and dissolved with $1.0 \mathrm{ml}$ of scintillation grade tissue solubilizer (Eastman Kodak, Rochester, NY) at $40^{\circ} \mathrm{C}$ for $24-48 \mathrm{~h}$. Samples were then decolorized by adding $0.5 \mathrm{ml}$ of $30^{\circ} \mathrm{c}$ hydrogen peroxide. Ten $\mathrm{ml}$ of aqueous scintillation cocktail (Amersham. Arlington Heights, IL) were added and the samples were stored in the dark for $24 \mathrm{~h}$ before being placed in a LS-9000 Beckman scintillation counter (Beckman Instruments, Irvine, CA).

In situ rat intestinal uptake experiments. using entrapped inulin, were performed to assess the ability of DSPC-CHOL liposomes to facilitate the oral uptake of entrapped drug as determined by its appearance in the urine. At the beginning of each experiment all urine was removed directly from the bladder by syringe. The entire urinary output during each experiment $(0.3-0.5 \mathrm{ml})$ was assayed by liquid scintillation counting. Four separate experiments were performed where the liposome suspensions were allowed to remain in the jejunum for $0.5,1.5,3.0$ and $3.5 \mathrm{~h}$ total.

\section{Tissue oxidation studies}

In situ uptake studies using washed DSPC-CHOL (2:1) liposomes with trace 
TABLE 1

HFFECT OF LIPID COMPOSITION ON LIPOSOMAL UPTAKE FROM THE RAT JEJUNL M IN SITU AT $37^{\circ} \mathrm{C}$.

\begin{tabular}{lllc}
\hline Lipid compusition & Number of experiments & $\begin{array}{l}k_{\mathrm{u}} \times 10^{4} / \mathrm{s} \\
\text { mean }( \pm \text { S.D. })\end{array}$ & $\begin{array}{l}\text { Half-life } \\
(\mathrm{min})\end{array}$ \\
\hline DMPC & 3 & $2.03(1.07)$ & 57 \\
DMPC-CHOL $(2: 1)$ & 4 & $2.24(0.64)$ & 52 \\
DPPC & 3 & $0.68(0.35)$ & 170 \\
DPPC-CHOL $(2: 1)$ & 3 & $2.61(0.86)$ & 44 \\
DSPC & 2 & $6.11(0.30)$ & 19 \\
DSPC-CHOL $(2: 1)$ & 6 & $5.61(0.42)$ & 21 \\
\hline
\end{tabular}

amounts of $\left[{ }^{3} \mathrm{H}\right] \mathrm{CHOL}$, and containing either $\left[{ }^{14} \mathrm{C}\right] \mathrm{PEG},\left[{ }^{14} \mathrm{C}\right] \mathrm{glucose}$, or $\mathrm{PBS}$ (empty liposomes) were performed. At the end of each experiment ( $30 \mathrm{~min}$ or $1 \mathrm{~h}$ ) the liposomal suspension was removed and the segment was rinsed vigorous y 3 times with 3-4 ml of PBS. or until the rinsings were devoid of significant amoun 15 of radioactivity. The rat was then sacrificed and the jejunal segment was remc ved immediately and stored below $0^{\circ} \mathrm{C}$.

A $10 \%(w / v)$ aqueous homogenate of each segment was prepared and three $1-\mathrm{ml}$ samples were dried in cellulose sample cups. The samples were oxidized in a Mc del 306 Packard Tricarb Sample Oxidizer (Packard Instruments, Downers Grove, L). with $99 \%$ recovery of both ${ }^{14} \mathrm{C}$ and ${ }^{3} \mathrm{H}$ as demonstrated by standardization pricedures. Each isotope was recovered separately. The ${ }^{14} \mathrm{C}\left(\mathrm{as} \mathrm{CO}_{2}\right)$ was trappec in Carbo-Sorb (Packard Instruments, Downers Grove, IL) with Permaflour (Pack ard Instruments. Downers Grove, IL) used as the scintillation cocktail and $\mathrm{H}$ (as $\mathrm{H}, \mathrm{O}$ ) was trapped and counted in Ready-Solv MP (Beckman Instruments. Irvine, CA;

\section{Results and Discussion}

\section{Effect of lipid composition}

The uptake of 'blank' or 'empty' liposomes of various lipid compositions from he rat jejunum, summarized in Table 1, exhibits apparent first-order kinetics. In all cases a plot of the log of the fraction remaining time was linear over the full $30 \mathrm{r}$ in of the experiment. The rate constant for uptake, $k_{u}$, is the slope of the above flot calculated by least-squares linear regression. Lipid concentrations in the liposo ne suspensions varied between 1.16 and $8.25 \mu \mathrm{mole} / \mathrm{ml}$. No correlation hetween upt: ke and lipid concentration was found for the liposomes tested. The size distribution of the liposome suspensions with identical lipid compositions should not be affected by the lipid concentration since all liposomes were prepared in the same manner, ut diluted to different degrees during gel chromatography. There is remarkably li tle variability in uptake for a given liposome composition since the liposomes were tot fractionated with respect to size and varied by almost one order of magnitude $u$ ith respect to lipid concentration. 
The Rate constant for uptake, $k_{u}$, is greatest for DSPC and DSPC-CHOL liposomes. The remaining lipid compositions exhibit similar rate constants except for DPPC, which had the lowest uptake of any lipid. The values of the phase transition ternperatures for DMPC, DPPC and DSPC were determined by differential scanning calorimetry to be $22.3,41.1$ and $56.7^{\circ} \mathrm{C}$, respectively. At the experimental temperature of $37^{\circ} \mathrm{C}$, DMPC liposomes wou?d exist in the liquid crystalline (fluid) state, while DSPC liposomes would exist in the gel (solid) state. At $37^{\circ} \mathrm{C}$, DPPC liposomes would be close to their transition temperature and virtually at the pre-transition peak temperature $\left(36-38^{\circ} \mathrm{C}\right)$ which corresponds to increased polar head group mobility (Ladbrooke et al., 1968). Liposomes are known to exhibit unique behavior near their phase transition temperatures, such as showing dramatic increases in permeability (de Gier et al., 1978).

Addition of at least 33 mole\% cholesterol to liposomal systems essentially eliminates the phase transition of the lecithin components (Hinz and Sturtevant, 1972). The inclusion of cholesterol to the DMPC or DSPC liposomes did not significantly alter their uptake profile. With DPPC liposomes, the addition of cholesterol increased the value of $k_{u}$, indicating a return of the bilayer to a more stable arrangement.

It should be pointed out that $k_{u}$ may represent numerous simultaneous liposomal uptake processes by the jejunum such as surface adsorption and transport. The values of $k_{~}$ do not differentiate these events.

In order to test for the possible occurrence of lipid exchange between liposomes and cell membranes in the jejunum, experiments were carried out with $\left[{ }^{14} \mathrm{C}\right]$ lecithins and $\left[{ }^{3} \mathrm{H}\right]$ cholesterol dual-labelled liposomes. The results, shown in Fig. 1, display an identical decrease for both markers indicating that lipid exchange is not significant

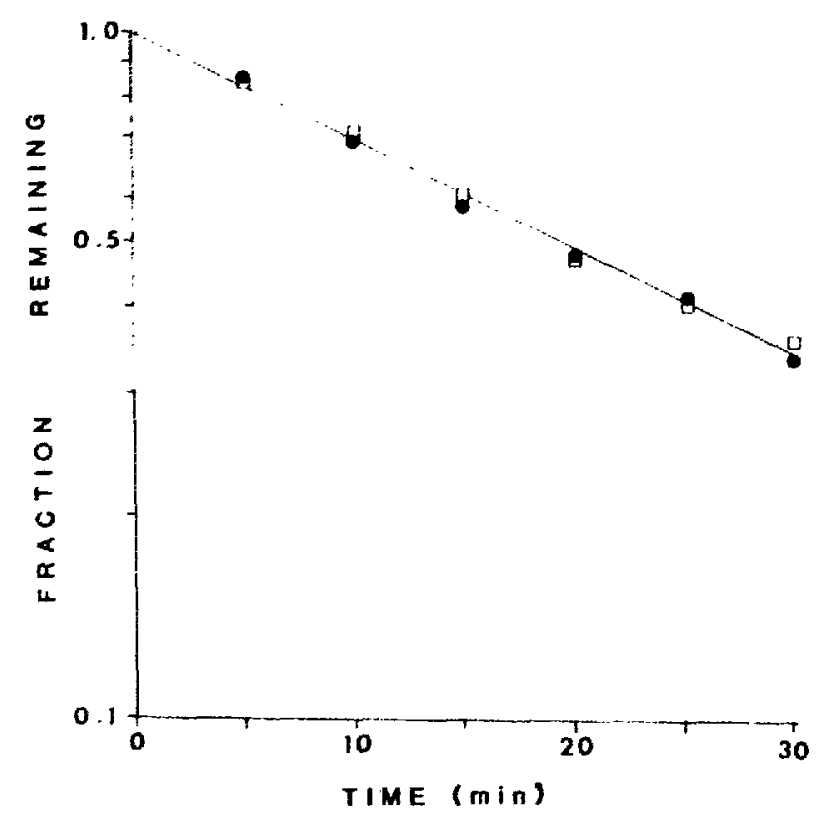

Fig. 1. Uptake of dual-lahelled DSPC-CHOL $(2: 1)$ liposomes from the rat jejunum in situ. 
during these uptake experiments. These results also strongly suggest that the uf take process involves intact liposomes.

\section{Tissue distribution studies}

The feasibility of using liposomes as an oral dosage form ultimately depends upon their ability to deliver compounds to the systemic circulation. Therefore. investigations of the fate of liposomal lipids and entrapped compounds following their in situ uptake were pursued. If liposomes are absorbed intact from the gut, as has seen proposed (Rowland and Woodley, 1981), their distribution should approximate that of parentally administered liposomes.

Since the $\mathrm{k}_{\mathrm{u}}$ values for DSPC-containing liposomes are greater than for the $\mathrm{cther}$ systems tested, and since Rowland and Woodley (1980) demonstrated that DMPC and DPPC liposomes, even in combination with cholesterol, are unstable in the gastrointestinal environment, all tissue distribution studies were limited to DSPC/CHOL liposomes.

Distribution studies were performed in order to determine whether liposomes or their lipid components coula be detected in various tissues. Radioactivity was fo and in only trace amounts in lymph and spleen (less than $0.5 \%$ of the absorbed dose), but was uniformly absent from liver samples. It is clear from this data that liposomal lipids are not able to gain access to either the lymphatic or general circulation in significant amounts under the conditions of these experiments. The absorptior of intact liposomes into the blood does not appear to be a possible explanation for the activity found in these samples. It is unlikely that liposomal uptake by the spl zen could occur in the absence of hepatic uptake since the mechanism is the same in both organs (Poste, 1983). Also, previous studies have consistently indicated that the liver is the primary site of recovery of liposomes from the general circulation (Gregoriadis et al., 1983). Furthermore, no evidence of inulin in the urine could be detected for the systems tested.

The results of these distribution studies provide valuable information on the ability of liposomes to act as an oral drug delivery system. DSPC-CHOL liposon zes appear to withstand gastric acidity and disruption by bile salts (Rowland and Woodley, 1980). The results of our in situ experiments indicate apparent uptake of these liposomes from the lumen of the jejunum. However, neither the liposoinal lipids nor entrapped, non-absorbable markers appear to be able to gain access to other tissues.

\section{Mass balance determination}

More detailed knowledge of the fat of liposomal lipids and entrapped compou ids can be acquired by investigating the recovery of radioactive markers (both lipid and entrapped compounil) from the jejunal segment. The method which proved to be most successful for intestinal tissue samples was tissue oxidation. The rest lts obtained by this method are shown in Fig. 2 .

A significant fraction of the suspension is retained by the jejunum desfite vigorous rinsing with buffer. The final rinse was determined to be free of activity thus indicating that the liposomes remaining in the jejunum may be adsorbed by 


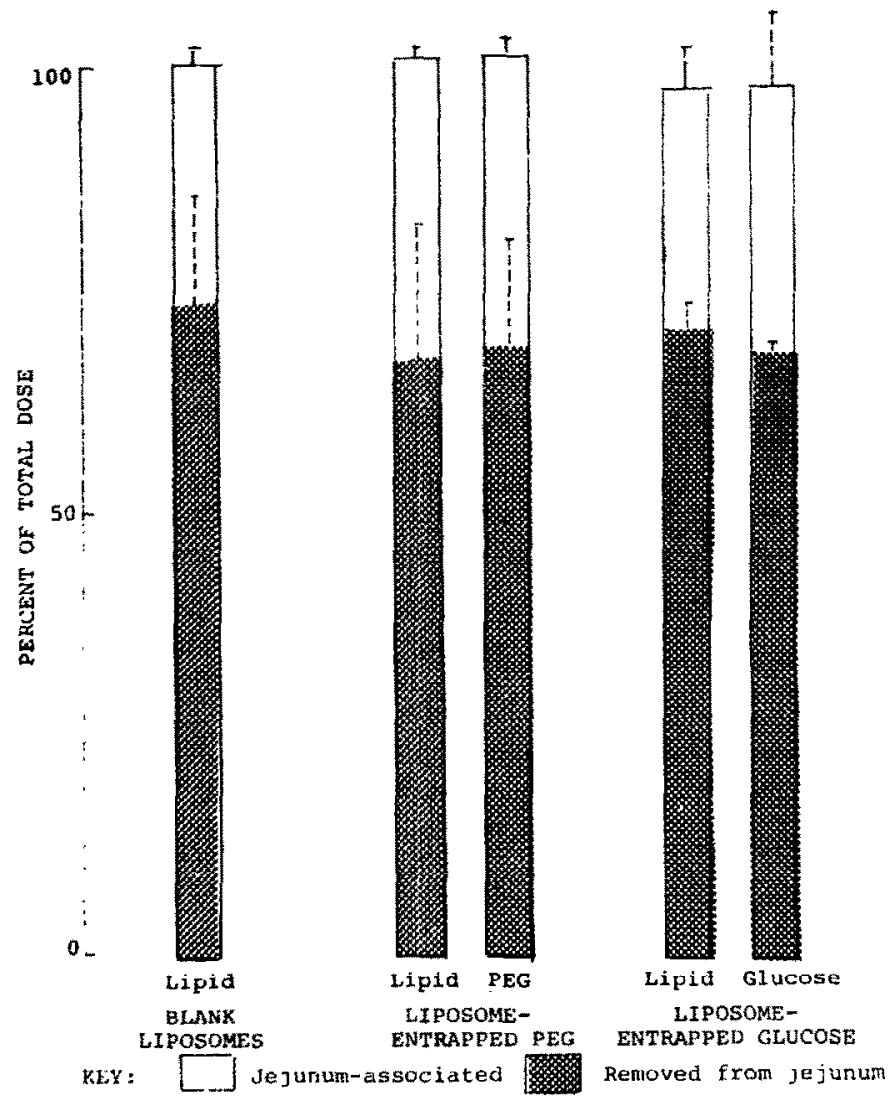

Fig. 2. Recovery of lipid and solute radiolabelled markers from the rat jejunum following in silu uptake experiments with DSPC.CHOL $(2: 1)$ liposomes.

some mechanism to the mucous or intestinal epithelium, or tightly adsorbed to the epithelial cell membrane. The partial recovery of radioactivity by rinsing suggests that a portion of the liposomes are taken up by a weak adsorption mechanism. Therefore, the uptake rate constants are a function of two or more mechanisms. It should be noted, that in all cases tested, mass balance was greater than $95 \%$.

The data from the entrapped glucose experiments suggest that the liposomes remain intact regardless of the mechanism by which they become associated with the jejunum. The almost full recovery of glucose strongly suggests that the fusion mechanism is not an important one, since if it was, glucose would be rapidly lost from the jejunum. The results also demonstrate that neither well-absorbed nor non-absorbed compounds can be effectively absorbed in entrapped form under these experimental conditions.

\section{Conclusions}

It may be concluded that in situ studies can provide a kinetic analysis of liposomal uptake from the jejunum. This technique allows access to other tissue compartments while maintaining control over a number of parameters at the 
absorption site. There is no evidence that liposomal lipids or entrapped mark rs can be transported from the jejunum to other compartments under the conditi ons of these experiments.

It should be noted that these experiments utilized the proximal end of the jejunum. However, digestion and absorption are sequential processes which occur over the entire length of the gastrointestinal tract. It is possible that in situ stu Jies of other segments of the tract, e.g. ileum, may produce results different than those presented here. Other variables, such as liposomal size and type (unilame lar is multilamellar) remain to be examined for their influence on the uptake proce is.

The studies presented here provide niethodologies and procedures to stu dy the obviously complex parameters influencing the effectiveness of liposomes as $\mathbf{i} \mathbf{n}$ oral drug delivery system.

\section{Acknowledgements}

Supported in part by gifts from Hoffmann-La Roche Inc.. Nutley. NI and Warner-Lambert Co.. Ann Arbor, MI.

\section{References}

de Gier, J., Blok. M.C.. van Dijck, R.W.M.. Mombers, C., Verkley. A.J.. van der Neut-Kok. E.C V. and van Deenen, L.L.M., Relations between lposomes and biomembranes. Ann. N.Y. Acau. S:i.. 308 (1978) 85-100.

Deshmukh. D.S. Bear. W.D. and Brocherhoff. H., Can intact lipasomes be absorhed in the gut? 1 fe Sci.. 28 (1981) $239-242$.

Gregoriadis, G. Kirby, C. and Senior, 1., Optimization of liposome behavior in vivo. Biol. (ell. 4* (1983) $11-18$.

Hinz. H.J. and Sturtevant, J.M., Calorimetric investigation of the influence of cholesterol on the tri nsition properties of bilayers formed from synthetic l-a-lecithins in aqueous suspension. J. Biol. Che $n$.. 247 (1972) 3698-3700.

Komiya. T., Park, J.Y., Kamani. A.. Ho. N.F.H. and Higuchi. W.I. Quantitative mechantstic st dies in simultaneous fluid flow and intestinal absorption using steroids as model solutes. Int. J. Ph urms. 4 (1980) 249-262.

Ladbrooke, B.D. Williams, R.M. and Chapman. D. Studies on lecithin-cholesterol-water intel sctwons by differential scanning calorimetry and X-ray diffraction. Biochim. Biophys. Acta. 150 (1968) 333-340.

Layton, D. Luckenbach, G.A., Andreesen, R. and Munder. P.G., The interaction of lipasomes wiln cells: the relation of cell specific toxicity to lipid compaxition. Eur. J. Cancer. 16 (19)0) 1529 1538

Miyamoto, V.K. and Stoeckenius, W.. Preparation and characteristics of lipid vesicles. 1. Membr. Biol. 4 (1971) $252-269$.

Pagano, R.E.. Schroit, A.J, and Struck. D.K.. Liposomes: from Physical Siructure to Thet upewite Applications, Elsevier/North-Holland. New York, 1981, pp. 323-348.

Patel, H.M. and Ryman, B.E., Oral administration of insulin by encapsulalion with lipmomes Ftas Lett. 62 (1976) 60-63.

Poste. G.. Liposome targeting in vivo: problems and opportunitics. Brol. Cell. 47 (19k3) 19-38.

Rowland, R.N. and Woodley, J.F. The stability of lipasomes in vitro lo pll. hule salis and pal steatic lipase. Biochim. Biophys. Acta. 620 (1980) 400-409.

Rowland, R.N. and Wondley, J.F., The uptake of distearoylphosphatidyleholine/cholesterol Irpor nes by rat intestinal sacs in vitro. Biochim. Biophys. Acta, 673 (1981) 217-223. 